\title{
Arterial Velocity Pulse Index as a Novel Marker of Atherosclerosis Using Pulse Wave Analysis on High Sensitivity Troponin T in Hypertensive Patients
}

\author{
Takashi Hitsumoto
}

\begin{abstract}
Background: The arterial velocity pulse index (AVI) is explored as a novel marker of atherosclerosis using pulse wave analysis in clinical settings. Recent clinical studies have reported that the level of high-sensitivity troponin $\mathrm{T}$ (hs-cTnT) is an important biomarker in hypertensive patients. The aim of this study was to clarify the impact of AVI on hs-cTnT in these patients.

Methods: This study enrolled 455 hypertensive outpatients (181 males and 274 females; mean age, $65 \pm 11$ years (mean \pm standard deviation)) without a history of cardiovascular events. AVI and hs-cTnT levels were measured using a commercial device, and relations among various clinical parameters, including AVI and hs-cTnT, were examined.
\end{abstract}

Results: Hs-cTnT was detected in 405 patients (89.0\%). AVI was significantly higher in patients with detectable hs-cTnT than in those without ( $28 \pm 7$ vs. $24 \pm 8$, respectively, $\mathrm{P}<0.001$ ). In patients with detectable hs-cTnT, there was a significant positive correlation between AVI and hs-cTnT $(r=0.42, \mathrm{P}<0.001)$. Furthermore, multiple regression analyses revealed that AVI was an independent variable when hscTnT was used as a subordinate factor. On the other hand, hs-cTnT age, Cornell electrocardiographic voltage, height, urinary albumin excretion, pulse rate, and derivatives of reactive oxygen metabolites test were independent variables when AVI was used as a subordinate factor.

Conclusion: The results of this study indicate that AVI reflects features of arterial wave reflection and is an important factor for hs-cTnT elevation in hypertensive patients.

Keywords: Arterial velocity pulse index; High-sensitivity troponin $\mathrm{T}$; Hypertension; Cardiovascular risk factor

\section{Introduction}

Blood pressure level is one of the most important target fac-

Manuscript accepted for publication April 05, 2017

Hitsumoto Medical Clinic, 2-7-7, Takezakicyou, Shimonoseki City, Yamaguchi 750-0025, Japan. Email: thitsu@jcom.home.ne.jp

doi: https://doi.org/10.14740/cr545w tors for preventing cardiovascular events in hypertensive patients. In recent years, reports have indicated the importance of pulse wave analysis, such as pulse wave velocity, cardio-ankle vascular index, and augmentation index. A number of studies have reported the clinical usefulness of these parameters as a marker of atherosclerosis in hypertensive patients [1-3]. Arterial velocity pulse index (AVI), a novel marker of atherosclerosis, uses oscillometric cuffs to evaluate pulse waveforms [4]. Increases in AVI reflect the enhancement of reflected waves resulting from atherosclerosis or other parameters. However, little is known about clinical significance of AVI because this parameter has only recently been explored and used in clinical settings.

Clinically, blood levels of high-sensitivity cardiac troponin $\mathrm{T}$ (hs-cTnT) represent a useful biomarker for evaluating the pathogenesis of heart failure and subclinical myocardial injury or predicting cardiovascular events [5-8]. Some clinical studies have reported that hs-cTnT elevation in hypertensive patients predicts incidents such as heart failure hospitalization, cardiac remodeling, and worsening of albuminuria [9-11]. Thus, from the perspective of primary prevention of cardiovascular events, hs-cTnT levels are an important factor in hypertensive patients.

To the best of our knowledge, there are no reports regarding the relationship between AVI and hs-cTnT. Therefore, this study attempts to clarify impact of hs-cTnT on AVI in hypertensive patients from the perspective of primary prevention of cardiovascular events. The authors also examine background factors in AVI.

\section{Materials and Methods}

\section{Patients}

Patients included in this study were enrolled between March 2014 and February 2016. The study population comprised 455 outpatients undergoing anti-hypertensive treatment. No patients in this study had a history of cardiovascular events such as ischemic heart disease, stroke, perivascular disease, persistent atrial fibrillation, or heart failure. There were 181 $(39.7 \%)$ males and $274(60.3 \%)$ females in this study, with a mean age of $65 \pm 11$ years (mean \pm standard deviation). All participants provided informed consent, and the study protocol was approved by the Local Ethics Committee. 


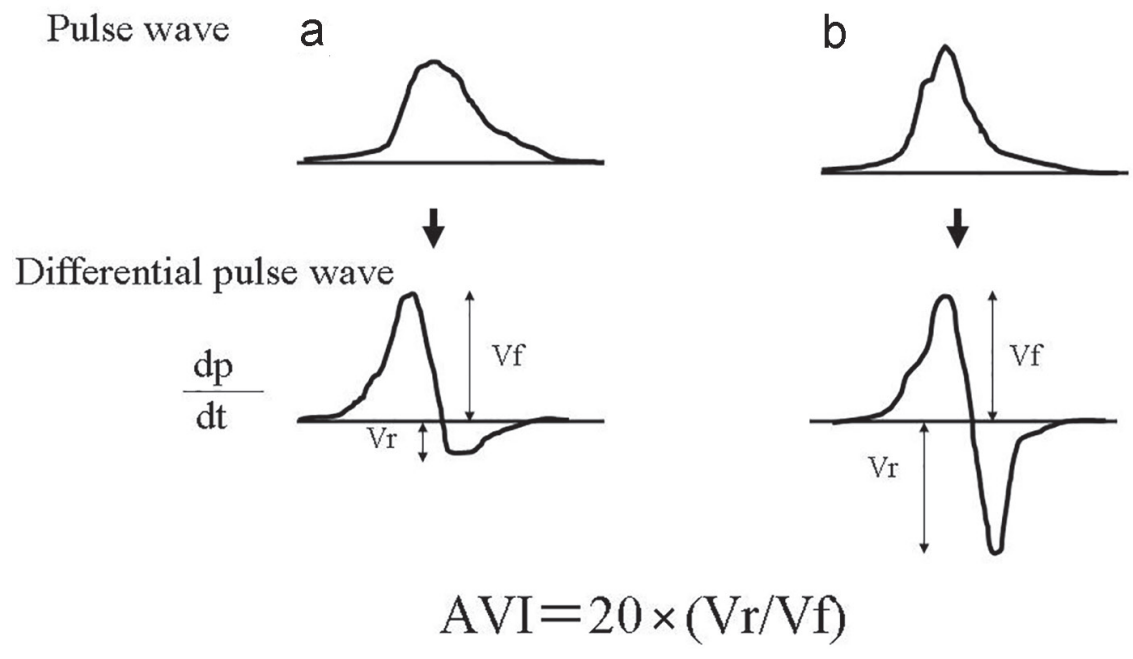

Figure 1. The measurement theory of AVI. (a) indicates normal pattern pulse wave pattern, and (b) indicates abnormal pulse wave pattern by increase of arterial reflection wave. $\mathrm{AVI}$ is automatically calculated as $20 \times(\mathrm{Vr} / \mathrm{Vf})$, wherein $\mathrm{Vf}$ is the first peak of the differentiated waveform between pulse wave and time and $\mathrm{Vr}$ is the absolute value of the bottom of the valley of differentiated waveforms between pulse wave and time. The systolic latter waveform rapidly increases and decreases in response to the reflected wave. However, the initial waveform is not influenced by the reflected wave. Vf reflects the initial waveform, and $\mathrm{Vr}$ reflects the decreased waveform. Therefore, as waves reflections increase, AVI increases in parallel with the increase in $\mathrm{Vr}$ unlike the normal pulse wave pattern. hs-cTnT: high-sensitivity cardiac troponin T; BP: blood pressure; FBG: fasting blood glucose; IRI: immunoreactive insulin; HOMA-IR: homeostatic model assessment of insulin resistance; LDL: low-density lipoprotein; HDL: high-density lipoprotein; eGFR: estimated glomerular filtration rate; BNP: brain natriuretic peptide; hs-CRP: high-sensitivity Creactive protein; d-ROMs: derivatives of reactive oxygen metabolites; $\mathrm{AVI}$ : arterial velocity pulse index; CCB: calcium channel blocker; RAS: renin-angiotensin system.

\section{Measurement of AVI}

AVI was measured using a commercial instrument (PASSESA AVI-1500; Shisei Datum, Tokyo, Japan), as previously described [4]. Measurements were taken in a quiet room with the temperature maintained at $20-25^{\circ} \mathrm{C}$. Treatment with antihypertensive drugs was stopped $24 \mathrm{~h}$ or more before measurements. The measurement theory of AVI is shown in Figure 1. AVI has the characteristics of a pulse wave pattern at higher cuff pressures compared with systolic blood pressure. Two variables were automatically measured: Vf and Vr. Consequently, AVI is automatically calculated as $20 \times(\mathrm{Vr} / \mathrm{Vf})$, wherein $\mathrm{Vf}$ is the first peak of the differentiated waveform between pulse wave and time and $\mathrm{Vr}$ is the absolute value of the bottom of the valley of differentiated waveforms between pulse wave and time. The systolic latter waveform rapidly increases and decreases in response to the reflected wave. However, the initial waveform is not influenced by the reflected wave. Vf reflects the initial waveform, and $\mathrm{Vr}$ reflects the decreased waveform. Therefore, as waves reflections increase, AVI increases in parallel with the increase in $\mathrm{Vr}$ unlike the normal pulse wave pattern. The validity and reliability of AVI measured using this method has been confirmed by previous reports [12].

\section{Estimation of cardiovascular risk factors}

Various clinical parameters, such as classic cardiovascular risk factors, left ventricular hypertrophy (LVH), insulin resistance, kidney function, brain natriuretic peptide (BNP) levels, inflammation, oxidative stress, and hs-cTnT levels, were evaluated. The degree of obesity was estimated using body mass index, which was calculated as weight $(\mathrm{kg})$ divided by height $\left(\mathrm{m}^{2}\right)$. Patients were considered positive for smoking if they habitually smoked cigarettes at the start of this study. Dyslipidemia was defined as a low-density lipoprotein cholesterol level greater than or equal to $140 \mathrm{mg} / \mathrm{dL}$, a high-density lipoprotein cholesterol level less than or equal to $40 \mathrm{mg} / \mathrm{dL}$, a triglyceride level greater than or equal to $150 \mathrm{mg} / \mathrm{dL}$, or based on an ongoing treatment for dyslipidemia. Diabetes mellitus was defined as a fasting blood glucose level greater than or equal to $126 \mathrm{mg} / \mathrm{dL}$ or based on an ongoing treatment for diabetes. The severity of LVH was evaluated by Cornell (R wave in $\mathrm{aVL}+\mathrm{S}$ wave in $\mathrm{V} 3$ ) electrocardiographic voltage calculations [13].

Blood samples were collected from the antecubital vein in the morning after $12 \mathrm{~h}$ of fasting. Total cholesterol and triglyceride levels were measured using standard enzymatic methods. Serum high-density lipoprotein cholesterol levels were measured by selective inhibition. Serum low-density lipoprotein cholesterol levels were calculated using the Friedewald equation [14]. Glucose and insulin levels were measured using the glucose oxidase method and an enzyme immunoassay, respectively. To measure insulin resistance, homeostatic model assessment of insulin resistance (HOMA-IR) was calculated as follows [15]: HOMA-IR = fasting glucose concentration $(\mathrm{mg} / \mathrm{dL}) \times$ fasting insulin concentration $(\mu \mathrm{g} / \mathrm{mL}) / 405$. The es- 


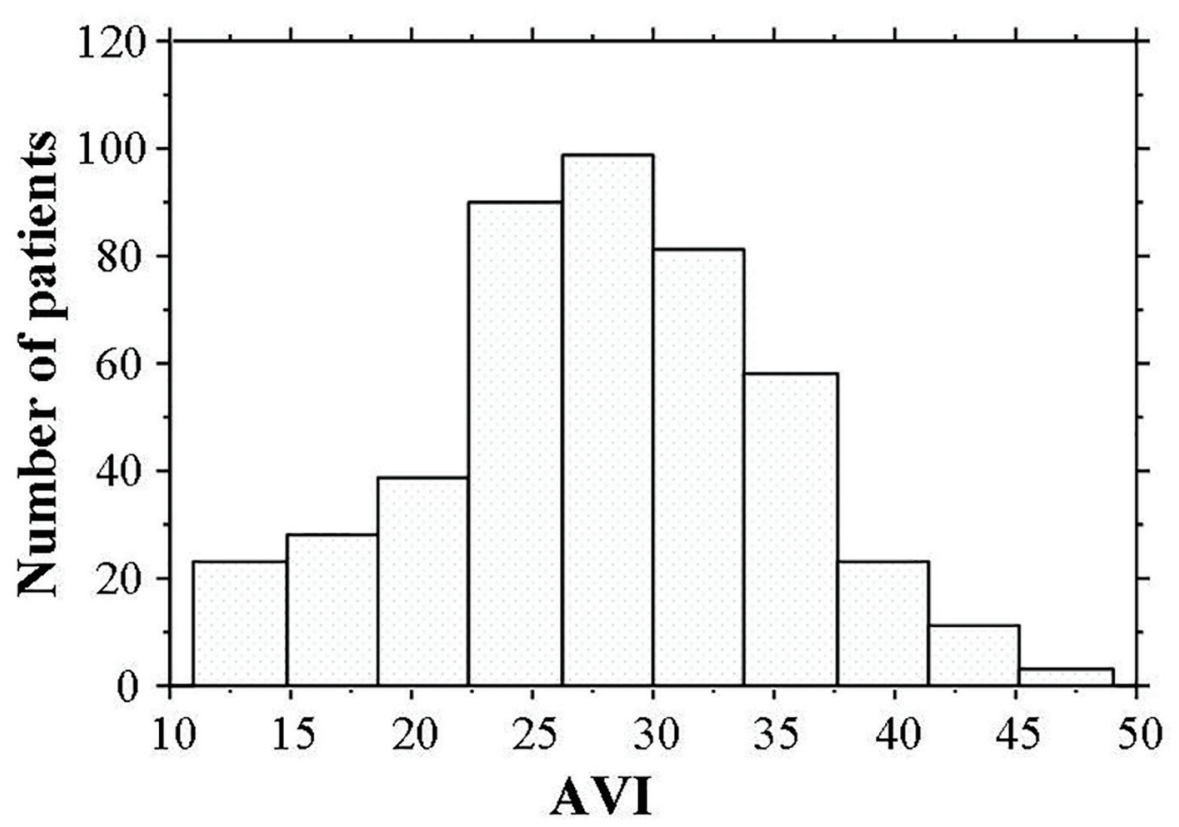

Figure 2. Histogram of AVI values. AVI ranged from 11 to 49 , and the median value was 28 . The distribution AVI was nearly normal. hs-cTnT: high-sensitivity cardiac troponin T; BP: blood pressure; FBG: fasting blood glucose; IRI: immunoreactive insulin; HOMA-IR: homeostatic model assessment of insulin resistance; LDL: low-density lipoprotein; HDL: high-density lipoprotein; eGFR: estimated glomerular filtration rate; BNP: brain natriuretic peptide; hs-CRP: high-sensitivity C-reactive protein; d-ROMs: derivatives of reactive oxygen metabolites; AVI: arterial velocity pulse index; $\mathrm{CCB}$ : calcium channel blocker; RAS: renin-angiotensin system.

timated glomerular filtration rate (eGFR) was calculated using the adjusted Modification of Diet in Renal Disease Study equation, which was proposed by the Working Group of the Japanese Chronic Kidney Disease Initiative [16], and urinary albumin concentration was measured using a commercial kit (Siemens/Bayer DCA 2000+ Analyzer; Siemens Healthineers, Tokyo, Japan). Blood levels of BNP were measured using a commercial kit (SHIONOSPOT Reader; Shionogi \& Co., Ltd, Osaka, Japan). High-sensitivity C-reactive protein (hs-CRP) levels were measured using high-sensitivity, latex-enhanced immunonephelometry. The reactive oxygen metabolites (dROMs) test, which reflects blood hydroperoxide concentrations, was performed using a commercial kit (Diacron; Grosseto, Italy) [17]. Hs-cTnT levels were also measured using a commercial kit (Roche Diagnostics, Switzerland) [18]. For the hs-cTnT assay, the lower limit of detection was $0.003 \mathrm{ng} / \mathrm{mL}$.

\section{Statistical analysis}

A commercially available statistical software program (Stat View-J 5.0; HULINKS Inc., Tokyo, Japan) was used for all statistical analyses. Data are expressed as the mean \pm standard deviation. Between-group comparisons were performed using the Student's t-test or Mann-Whitney U test, and the correlation coefficient was estimated using the Spearman rank-order correlation analysis. To clarify which independent factors contributed to increased hs-cTnT levels or AVI, multiple regression analysis was performed using hs-cTnT or AVI as a subordinate factor. A P value of less than 0.05 was considered statistically significant.

\section{Results}

Distribution of AVI (Fig. 2) ranged from 11 to 49, the median value was 28 , and the distribution AVI was nearly normal. Patient characteristics are presented in Table 1. Hs-cTnT was detected in $405(89.0 \%)$ patients. Age, body weight, body mass index, Cornell electrocardiographic voltage, urinary albumin excretion BNP levels, and d-ROMs test were significantly higher and eGFR was significantly lower in patients with detectable hs-cTnT. AVI was significantly higher in patients with detectable hs-cTnT than in those with undetectable hs-cTnT. Table 2 shows correlations between hs-cTnT levels, AVI, and various clinical parameters in the cohort with detectable hs-cTnT. Age, body mass index, Cornell electrocardiographic voltage, eGFR, urinary albumin excretion, BNP levels, hs-CRP levels, d-ROMs test, and AVI were all significantly correlated with hs-cTnT levels. Sex differences, age, height, body weight, systolic blood pressure, pulse rate, Cornell electrocardiographic voltage, eGFR, urinary albumin excretion, BNP levels, hs-CRP levels, d-ROMs test, and hs-cTnT were significantly correlated with AVI.

Multiple regression analyses for hs-cTnT or AVI as a subordinate factor were performed with explanatory variables that were significant during univariate analysis. AVI, age, BNP levels, urinary albumin excretion, d-ROMs test, and Cornell 
Table 1. Patient Characteristics

\begin{tabular}{|c|c|c|c|c|}
\hline & Overall & hs-cTnT non-detection & hs-cTnT detection & P value \\
\hline $\mathrm{n}$ (male/female) & $455(181 / 274)$ & $50(20 / 30)$ & $405(161 / 244)$ & 0.973 \\
\hline Age (years) & $65 \pm 11$ & $61 \pm 11$ & $66 \pm 11$ & $<0.01$ \\
\hline Height (m) & $1.6 \pm 0.1$ & $1.6 \pm 0.1$ & $1.6 \pm 0.1$ & 0.873 \\
\hline Body weight (kg) & $57 \pm 10$ & $54 \pm 11$ & $58 \pm 11$ & $<0.05$ \\
\hline Body mass index $\left(\mathrm{kg} / \mathrm{m}^{2}\right)$ & $22 \pm 4$ & $21 \pm 3$ & $23 \pm 4$ & $<0.05$ \\
\hline Smoking, n (\%) & $109(24)$ & $13(26)$ & $96(24)$ & 0.720 \\
\hline Systolic BP (mm Hg) & $157 \pm 8$ & $157 \pm 10$ & $157 \pm 8$ & 0.953 \\
\hline Diastolic BP (mm Hg) & $91 \pm 9$ & $91 \pm 9$ & $91 \pm 10$ & 0.572 \\
\hline Pulse rate $(\mathrm{mm} \mathrm{Hg})$ & $68 \pm 11$ & $70 \pm 11$ & $68 \pm 10$ & 0.129 \\
\hline Cornell voltage (mm) & $15 \pm 6$ & $12 \pm 7$ & $15 \pm 5$ & $<0.001$ \\
\hline Diabetes mellitus (\%) & $189(42)$ & $20(40)$ & $169(42)$ & 0.816 \\
\hline FBG (mg/dL) & $113 \pm 24$ & $112 \pm 20$ & $113 \pm 24$ & 0.819 \\
\hline IRI $(\mu \mathrm{g} / \mathrm{mL})$ & $7.3 \pm 4.4$ & $7.5 \pm 4.8$ & $7.3 \pm 4.4$ & 0.626 \\
\hline HOMA-IR & $2.1 \pm 1.4$ & $2.1 \pm 1.3$ & $2.1 \pm 1.4$ & 0.864 \\
\hline Dyslipidemia (\%) & $346(76)$ & $29(54)$ & $317(64)$ & 0.172 \\
\hline Total cholesterol (mg/dL) & $221 \pm 42$ & $219 \pm 42$ & $221 \pm 41$ & 0.541 \\
\hline LDL cholesterol (mg/dL) & $137 \pm 37$ & $136 \pm 40$ & $137 \pm 38$ & 0.658 \\
\hline Triglyceride (mg/dL) & $177 \pm 72$ & $170 \pm 83$ & $179 \pm 69$ & 0.279 \\
\hline HDL cholesterol (mg/dL) & $49 \pm 16$ & $49 \pm 14$ & $48 \pm 16$ & 0.62 \\
\hline $\mathrm{eGFR}\left(\mathrm{mL} / \mathrm{min} / 1.73 \mathrm{~m}^{2}\right)$ & $64 \pm 21$ & $69 \pm 21$ & $63 \pm 18$ & $<0.05$ \\
\hline Log-urinary albumin (mg/gCr) & $1.5 \pm 0.5$ & $1.3 \pm 0.5$ & $1.6 \pm 0.5$ & $<0.01$ \\
\hline $\log -\mathrm{BNP}(\mathrm{pg} / \mathrm{mL})$ & $86 \pm 67$ & $57 \pm 55$ & $89 \pm 67$ & $<0.01$ \\
\hline Log-hs-CRP (mg/L) & $-1.2 \pm 0.6$ & $-1.2 \pm 0.6$ & $-1.2 \pm 0.6$ & 0.331 \\
\hline d-ROMs test (U. Carr) & $355 \pm 93$ & $330 \pm 97$ & $359 \pm 92$ & $<0.01$ \\
\hline AVI & $28 \pm 7$ & $24 \pm 8$ & $28 \pm 7$ & $<0.001$ \\
\hline Log-hs-cTnT (ng/mL) & $-1.9 \pm 0.2$ & - & $-1.9 \pm 0.2$ & - \\
\hline \multicolumn{5}{|l|}{ Medication } \\
\hline $\mathrm{CCB}, \mathrm{n}(\%)$ & $332(73)$ & $36(72)$ & $296(73)$ & 0.652 \\
\hline RAS inhibitor, n (\%) & $223(49)$ & $25(50)$ & $198(49)$ & 0.753 \\
\hline Statin, n (\%) & $155(34)$ & $17(34)$ & $138(34)$ & 0.889 \\
\hline
\end{tabular}

Data are expressed as mean \pm SD. hs-cTnT: high-sensitivity cardiac troponin T; BP: blood pressure; FBG: fasting blood glucose; IRI: immunoreactive insulin; HOMA-IR: homeostatic model assessment of insulin resistance; LDL: low-density lipoprotein; HDL: high-density lipoprotein; eGFR: estimated glomerular filtration rate; BNP: brain natriuretic peptide; hs-CRP: high-sensitivity C-reactive protein; d-ROMs: derivatives of reactive oxygen metabolites; AVI: arterial velocity pulse index; CCB: calcium channel blocker; RAS: renin-angiotensin system.

electrocardiographic voltage were independent variables for hs-cTnT. Hs-cTnT, age, Cornell electrocardiographic voltage, height, urinary albumin excretion, pulse rate, and d-ROMs test were independent variables for AVI (Table 3).

\section{Discussion}

Arterial wave reflection is affected by aging, sex differences, height, and pulse rate [19-21]. The results of this study also indicated that these parameters were significantly associated with AVI. Furthermore, age, height, and pulse rate were independent variables for AVI. Thus, AVI also reflects features of arterial wave reflection. The significant relationship between AVI and hs-cTnT, as determined by multivariate analysis, indicates that arterial wave reflection is an important factor for the progression of subclinical myocardial damage in hypertensive patients. The augmentation index is also a useful marker of arterial wave reflection, and an augmentation index for the radial or carotid artery can be non-invasively measured by tonometry. Augmentation indices from tonometry are important markers of arterial wave reflection or cardiovascular risk fac- 
Table 2. Correlation Between hs-cTnT, AVI and Clinical Parameters in hs-cTnT Detectable Patients

\begin{tabular}{|c|c|c|}
\hline & $\mathbf{r}$ & \\
\hline & Log-hs-cTnT & AVI \\
\hline Sex $($ female $=0$, male $=1)$ & -0.06 & $-0.13 * *$ \\
\hline Age & $0.40^{*}$ & $0.36^{*}$ \\
\hline Height & 0.06 & $-0.32 *$ \\
\hline Body weight & 0.09 & $-0.18^{*}$ \\
\hline Body mass index & $0.10 * * *$ & -0.08 \\
\hline Smoking $($ no $=0$, yes $=1)$ & 0.02 & 0.09 \\
\hline Systolic BP & 0.08 & $0.12 * *$ \\
\hline Diastolic BP & 0.05 & 0.08 \\
\hline Pulse rate & 0.04 & $0.26^{*}$ \\
\hline Cornell voltage & $0.21 *$ & $0.35^{*}$ \\
\hline Diabetes mellitus (no $=0$, yes $=1$ ) & 0.07 & 0.08 \\
\hline FBG & 0.03 & 0.09 \\
\hline IRI & 0.04 & 0.07 \\
\hline Log-HOMA-IR & 0.06 & 0.06 \\
\hline Dyslipidemia $($ no $=0$, yes $=1)$ & -0.03 & -0.01 \\
\hline Total cholesterol & -0.06 & 0.05 \\
\hline LDL cholesterol & -0.05 & 0.04 \\
\hline Triglyceride & -0.03 & 0.06 \\
\hline HDL cholesterol & -0.06 & -0.04 \\
\hline eGFR & $-0.13^{* *}$ & $-0.10 * * *$ \\
\hline Log-urinary albumin & $0.20^{*}$ & $0.29 *$ \\
\hline Log-BNP & $0.25^{*}$ & $0.35 * * *$ \\
\hline Log-hs-CRP & $0.10 * * *$ & $0.11 * * *$ \\
\hline d-ROMs test & $0.35^{*}$ & $0.37 *$ \\
\hline AVI & $0.42 *$ & - \\
\hline Log-hs-cTnT & - & $0.42 *$ \\
\hline $\mathrm{CCB}($ no $=0$, yes $=1)$ & -0.06 & -0.05 \\
\hline RAS inhibitor $($ no $=0$, yes $=1)$ & -0.06 & -0.05 \\
\hline Statin $($ no $=0$, yes $=1)$ & -0.03 & -0.02 \\
\hline
\end{tabular}

$r$ is correlation coefficient. ${ }^{*} P<0.001$, ${ }^{* *} P<0.01$, ${ }^{* * *} P<0.05$. hs-cTnT: high-sensitivity cardiac troponin T; BP: blood pressure; FBG: fasting blood glucose; IRI: immunoreactive insulin; HOMA-IR: homeostatic model assessment of insulin resistance; LDL: low-density lipoprotein; HDL: high-density lipoprotein; eGFR: estimated glomerular filtration rate; BNP: brain natriuretic peptide; hs-CRP: high-sensitivity C-reactive protein; d-ROMs: derivatives of reactive oxygen metabolites; AVI: arterial velocity pulse index; CCB: calcium channel blocker; RAS: reninangiotensin system.

tors [2, 22]. However, for the precise measurement of an arterial augmentation index, expertize is required. Conversely, AVI can be automatically calculated using conventional blood pressure measurements. Thus, AVI measurements have procedural advantages over augmentation index measurements. Sasaki-Nakashima et al reported that AVI had a significant re-
Table 3. Multiple Regression Analysis

\begin{tabular}{|llll}
\hline Dependent variable & $\boldsymbol{\beta}$ & t value & P value \\
\hline (A) AVI & 0.35 & 6.6 & $<0.001$ \\
\hline Age & 0.33 & 6.0 & $<0.001$ \\
BNP & 0.14 & 3.0 & $<0.01$ \\
Log-urinary albumin & 0.12 & 2.5 & $<0.01$ \\
d-ROMs test & 0.11 & 2.3 & $<0.01$ \\
Cornell voltage & 0.10 & 2.1 & $<0.05$ \\
eGFR & -0.07 & -1.5 & 0.152 \\
Log-hs-CRP & 0.06 & 1.4 & 0.148 \\
Body mass index & 0.02 & 0.3 & 0.730 \\
(B) Log-hs-cTnT & 0.27 & 6.3 & $<0.001$ \\
Age & 0.25 & 6.0 & $<0.001$ \\
Cornell voltage & 0.23 & 5.6 & $<0.001$ \\
Height & -0.22 & -3.6 & $<0.001$ \\
Log-urinary albumin & 0.12 & 2.9 & $<0.01$ \\
Pulse rate & -0.11 & -2.8 & $<0.01$ \\
d-ROMs test & 0.11 & 2.7 & $<0.01$ \\
BNP & 0.09 & 1.9 & 0.063 \\
Sex & 0.09 & 1.8 & 0.132 \\
Systolic BP & 0.06 & 1.5 & 0.168 \\
Log-hs-CRP & 0.06 & 1.4 & 0.173 \\
eGFR & -0.04 & -1.2 & 0.289 \\
Body weight & -0.09 & -1.9 & 0.058 \\
\hline
\end{tabular}

(A) Subordinate factor is log-hs-cTnT. $\mathrm{R}^{2}=0.23, \mathrm{~F}=12.5, \mathrm{P}<0.001$. (B) Subordinate factor is AVI. $\mathrm{R}^{2}=0.43, \mathrm{~F}=22.7, \mathrm{P}<0.001 .$. hs-cTnT: high-sensitivity cardiac troponin T; BP: blood pressure; FBG: fasting blood glucose; IRI: immunoreactive insulin; HOMA-IR: homeostatic model assessment of insulin resistance; LDL: low-density lipoprotein; HDL: high-density lipoprotein; eGFR: estimated glomerular filtration rate; BNP: brain natriuretic peptide; hs-CRP: high-sensitivity C-reactive protein; d-ROMs: derivatives of reactive oxygen metabolites; AVI: arterial velocity pulse index; $\mathrm{CCB}$ : calcium channel blocker; RAS: reninangiotensin system.

lationship with the radial augmentation index [12]. However, the correlation coefficient for an association between AVI and augmentation index is weak $(\mathrm{r}=0.207)$. Therefore, AVI differs from the augmentation index, although both parameters reflect the arterial wave reflection. Further studies are required for the use of AVI as a significance measure of arterial wave reflection compared with the augmentation index.

Pulse wave velocity increases relative to increases in arterial wall stiffness, and some clinical studies have indicated a significant relationship between pulse wave velocity and subclinical myocardial damage using troponin or serum hearttype fatty acid-binding proteins [23-25]. Furthermore, arterial wall stiffness is an important factor for increasing the arterial wave reflection. Okamoto et al reported that AVI had a significant relationship with the cardio-ankle vascular index, which is a method for evaluating of pulse wave velocity [26]. Sev- 
eral studies have indicated that left ventricular dysfunction is significantly associated with myocardial damage [27, 28]; furthermore, increases in aortic artery stiffness cause left ventricular dysfunction [29]. Therefore, we hypothesize that the relationship between hs-cTnT and AVI identified in this study reflects myocardial damage via left ventricular dysfunction resulting from an increase in vascular resistance or afterload.

Oxidative stress is closely associated with heart failure progression or vascular events. Several pathways in which oxidative stress leads to myocardial injury have been identified, including dysfunction of the mitochondrial electron transport complex, nicotinamide adenine dinucleotide phosphate oxidase activity, and myocardial cell apoptosis $[30,31]$. This study revealed the importance of oxidative stress in myocardial injury due to hypertension and during the subclinical stages of heart failure. In addition, clinical studies have shown a significant association between arterial wave reflection and oxidative stress $[32,33]$. In this study, multivariate analysis revealed a significant correlation between the d-ROMs test as a marker of oxidative stress in vivo and AVI or hs-cTnT, suggesting that oxidative stress affects both myocardial injury and arterial wave reflection in hypertensive patients, further causing cardiovascular events. Therefore, anti-oxidant treatment may be an important therapy for the prevention of cardiovascular events by protecting both cardiomyocyte cells and arterial vessel walls.

In this study, urinary albumin concentration was independently associated with both hs-cTnT and AVI. The urinary albumin concentration reflects glomerular hypertension and systemic endothelial function [34]. Endothelial dysfunction is the first step in the progression to atherosclerosis and an important factor for the elevation of troponin $\mathrm{T}[35,36]$. The relationship between arterial wave reflection and endothelial function is controversial. Researchers have reported a significant relationship between the augmentation index and endothelial function, which was estimated by flow-mediated vasodilation in the brachial artery or albuminuria [32, 37]. Conversely, Sasaki-Nakashima et al reported that AVI had no significant relationship with flow-mediated vasodilation in the brachial artery [12]. Differences between this study and Sasaki-Nakashima's reports concerning relationship between AVI and endothelial function may be due to differences in the methods of evaluating the endothelial function or sample size. However, the results of this study indicate that AVI may reflect systemic endothelial function. Thus, increases in AVI as a causative factor for elevation of hs-cTnT in hypertensive patients may be partially explained by endothelial dysfunction.

Researchers have also reported that LVH is significantly associated with hs-cTnT levels in hypertensive patients $[6,38]$. The results of this study are consistent with previous reports. LVH was also selected as an independent variable for AVI. However, Hashimoto et al reported that augmentation index at the radial artery had a significant relationship with $\mathrm{LVH}$, as assessed using ultrasound in hypertensive patients [39]. LVH is an important factor for the prognosis of these patients. Therefore, it may be possible to inhibit the progression of LVH in them by adequate control of blood pressure levels with a lower AVI value, which would consequently improve their prognosis.

\section{Limitations}

This study has several limitations. First, treatment with antihypertensive drugs was stopped $24 \mathrm{~h}$ or more before measurement to avoid influencing AVI. However, $24 \mathrm{~h}$ was not sufficient to mitigate the effects of long-acting drugs such as amlodipine. Second, ultrasonic echocardiography, coronary angiography, and multidetector computed tomography angiography were not performed. Therefore, cardiovascular diseases such as heart failure or coronary artery disease may have gone undetected. Third, medical treatments using anti-oxidant drugs such as calcium channel blockers, renin-angiotensin receptor inhibitors, or statins may have influenced the study results. However, the study results indicate that the effect of these drugs is insignificant. Finally, this study was cross-sectional in a single unit and the sample size was relatively small. Additional prospective studies, including evaluations of interventional therapies, are required to clarify the clinical significance of AVI as a risk factor for cardiovascular disease in hypertensive patients.

\section{Conclusions}

This study evaluated the impact of AVI as novel marker of atherosclerosis using pulse wave analysis on hs-cTnT in hypertensive patients. The results of this study indicated that AVI reflects features of arterial wave reflection and is an important factor for hs-cTnT elevation in these patients.

\section{Competing Interests}

Author has no competing interests.

\section{Grant Support}

None.

\section{Financial Disclosure}

None.

\section{References}

1. Zheng M, Huo Y, Wang X, Xu X, Qin X, Tang G, Xing H, et al. A prospective study on pulse wave velocity (PWV) and response to anti-hypertensive treatments: PWV determines BP control. Int J Cardiol. 2015;178:226-231.

2. Williams B, Lacy PS, Thom SM, Cruickshank K, Stanton A, Collier D, Hughes AD, et al. Differential impact of blood pressure-lowering drugs on central aortic pressure and clinical outcomes: principal results of the Conduit Artery Function Evaluation (CAFE) study. Circulation. 2006;113(9):1213-1225. 
3. Kubozono T, Ohishi M. Prognostic Significance of Regional Arterial Stiffness for Stroke in Hypertension. Pulse (Basel). 2015;3(2):98-105.

4. Sueta D, Yamamoto E, Tanaka T, Hirata Y, Sakamoto K, Tsujita K, Kojima S, et al. The accuracy of central blood pressure waveform by novel mathematical transformation of non-invasive measurement. Int $\mathrm{J}$ Cardiol. 2015;189:244-246.

5. Latini R, Masson S, Anand IS, Missov E, Carlson M, Vago T, Angelici L, et al. Prognostic value of very low plasma concentrations of troponin $\mathrm{T}$ in patients with stable chronic heart failure. Circulation. 2007;116(11):12421249.

6. Sato Y, Yamamoto E, Sawa T, Toda K, Hara T, Iwasaki T, Fujiwara H, et al. High-sensitivity cardiac troponin $\mathrm{T}$ in essential hypertension. J Cardiol. 2011;58(3):226-231.

7. Saunders JT, Nambi V, de Lemos JA, Chambless LE, Virani SS, Boerwinkle E, Hoogeveen RC, et al. Cardiac troponin $\mathrm{T}$ measured by a highly sensitive assay predicts coronary heart disease, heart failure, and mortality in the Atherosclerosis Risk in Communities Study. Circulation. 2011;123(13):1367-1376.

8. Otsuka T, Kawada T, Ibuki C, Seino Y. Association between high-sensitivity cardiac troponin $\mathrm{T}$ levels and the predicted cardiovascular risk in middle-aged men without overt cardiovascular disease. Am Heart J. 2010;159(6):972-978.

9. Pokharel Y, Sun W, de Lemos JA, Taffet GE, Virani SS, Ndumele CE, Mosley TH, et al. High-sensitivity troponin $\mathrm{T}$ and cardiovascular events in systolic blood pressure categories: atherosclerosis risk in communities study. Hypertension. 2015;65(1):78-84.

10. Miao DM, Zhang LP, Yu HP, Zhang JY, Xiao WK, Ye P. Serum levels of high-sensitivity troponin T: a novel marker for left ventricular remodeling and performance in hypertensive subjects. Genet Mol Res. 2014;13(3):51435153.

11. Hellemons ME, Lambers Heerspink HJ, Gansevoort RT, de Zeeuw D, Bakker SJ. High-sensitivity troponin T predicts worsening of albuminuria in hypertension; results of a nested case-control study with confirmation in diabetes. J Hypertens. 2013;31(4):805-812.

12. Sasaki-Nakashima R, Kino T, Chen L, Doi H, Minegishi S, Abe K, Sugano T, et al. Successful prediction of cardiovascular risk by new non-invasive vascular indexes using suprasystolic cuff oscillometric waveform analysis. J Cardiol. 2017;69(1):30-37.

13. Okin PM, Devereux RB, Liu JE, Oikarinen L, Jern S, Kjeldsen SE, Julius S, et al. Regression of electrocardiographic left ventricular hypertrophy predicts regression of echocardiographic left ventricular mass: the LIFE study. J Hum Hypertens. 2004;18(6):403-409.

14. Friedewald WT, Levy RI, Fredrickson DS. Estimation of the concentration of low-density lipoprotein cholesterol in plasma, without use of the preparative ultracentrifuge. Clin Chem. 1972;18(6):499-502.

15. Matthews DR, Hosker JP, Rudenski AS, Naylor BA, Treacher DF, Turner RC. Homeostasis model assessment: insulin resistance and beta-cell function from fasting plasma glucose and insulin concentrations in man. Diabetologia. 1985;28(7):412-419.

16. Imai $\mathrm{E}$, Horio $\mathrm{M}$, Nitta $\mathrm{K}$, Yamagata $\mathrm{K}$, Iseki $\mathrm{K}$, Hara $\mathrm{S}$, Ura N, et al. Estimation of glomerular filtration rate by the MDRD study equation modified for Japanese patients with chronic kidney disease. Clin Exp Nephrol. 2007;11(1):41-50.

17. Cesarone MR, Belcaro G, Carratelli M, Cornelli U, De Sanctis MT, Incandela L, Barsotti A, et al. A simple test to monitor oxidative stress. Int Angiol. 1999;18(2):127-130.

18. Mingels A, Jacobs L, Michielsen E, Swaanenburg J, Wodzig W, van Dieijen-Visser M. Reference population and marathon runner sera assessed by highly sensitive cardiac troponin $\mathrm{T}$ and commercial cardiac troponin $\mathrm{T}$ and I assays. Clin Chem. 2009;55(1):101-108.

19. Hayward CS, Kelly RP. Gender-related differences in the central arterial pressure waveform. J Am Coll Cardiol. 1997;30(7):1863-1871.

20. Smulyan H, Marchais SJ, Pannier B, Guerin AP, Safar ME, London GM. Influence of body height on pulsatile arterial hemodynamic data. J Am Coll Cardiol. 1998;31(5):1103-1109.

21. Wilkinson IB, MacCallum H, Flint L, Cockcroft JR, Newby DE, Webb DJ. The influence of heart rate on augmentation index and central arterial pressure in humans. J Physiol. 2000;525(Pt 1):263-270.

22. Hitsumoto T. Factors Associated with High-sensitivity Cardiac Troponin T in Patients with Type 2 Diabetes Mellitus. J Nippon Med Sch. 2015;82(6):274-280.

23. Bai Y, Ye P, Luo L, Xiao W, Xu R, Wu H, Bai J. Arterial stiffness is associated with minimally elevated high-sensitivity cardiac, troponin T levels in a community-dwelling population. Atherosclerosis. 2011;218(2):493-498.

24. Gedikli O, Ozturk S, Yilmaz H, Baykan M, Kiris A, Durmus I, Baltaci D, et al. Relationship between arterial stiffness and myocardial damage in patients with newly diagnosed essential hypertension. Am J Hypertens. 2008;21(9):989-993.

25. Hitsumoto T, Shirai K. Factors affecting high-sensitivity cardiac troponin T elevation in Japanese metabolic syndrome patients. Diabetes Metab Syndr Obes. 2015;8:157162.

26. Okamoto M, Nakamura F, Musha T, Kobayashi Y. Association between novel arterial stiffness indices and risk factors of cardiovascular disease. BMC Cardiovasc Disord. 2016;16(1):211.

27. Jungbauer CG, Riedlinger J, Buchner S, Birner C, Resch M, Lubnow M, Debl K, et al. High-sensitive troponin T in chronic heart failure correlates with severity of symptoms, left ventricular dysfunction and prognosis independently from N-terminal pro-b-type natriuretic peptide. Clin Chem Lab Med. 2011;49(11):1899-1906.

28. Buiten MS, de Bie MK, Rotmans JI, Dekker FW, van Buren M, Rabelink TJ, Cobbaert CM, et al. Serum Cardiac Troponin-I is Superior to Troponin-T as a Marker for Left Ventricular Dysfunction in Clinically Stable Patients with End-Stage Renal Disease. PLoS One. 2015;10(8):e0134245.

29. Jaroch J, Loboz Grudzien K, Bociaga Z, Kowalska A, 
Kruszynska E, Wilczynska M, Dudek K. The relationship of carotid arterial stiffness to left ventricular diastolic dysfunction in untreated hypertension. Kardiol Pol. 2012;70(3):223-231.

30. Li SY, Yang X, Ceylan-Isik AF, Du M, Sreejayan N, Ren J. Cardiac contractile dysfunction in Lep/Lep obesity is accompanied by NADPH oxidase activation, oxidative modification of sarco(endo)plasmic reticulum Ca2+-ATPase and myosin heavy chain isozyme switch. Diabetologia. 2006;49(6):1434-1446.

31. Boudina S, Abel ED. Diabetic cardiomyopathy, causes and effects. Rev Endocr Metab Disord. 2010;11(1):31-39.

32. Hitsumoto T. Clinical significance of the augmentation index in patients with preserved kidney function. J Nippon Med Sch. 2012;79(6):422-429.

33. Tanindi A, Erkan AF, Alhan A, Tore HF. Arterial stiffness and central arterial wave reflection are associated with serum uric acid, total bilirubin, and neutrophil-to-lymphocyte ratio in patients with coronary artery disease. Anatol J Cardiol. 2015;15(5):396-403.

34. Stehouwer CD, Nauta JJ, Zeldenrust GC, Hackeng WH, Donker AJ, den Ottolander GJ. Urinary albumin excretion, cardiovascular disease, and endothelial dysfunc- tion in non-insulin-dependent diabetes mellitus. Lancet. 1992;340(8815):319-323.

35. Lai S, Dimko M, Galani A, Coppola B, Innico G, Frassetti N, Mazzei ED, et al. Early markers of cardiovascular risk in chronic kidney disease. Ren Fail. 2015;37(2):254261.

36. Xiao W, Ye P, Cao R, Yang X, Bai Y, Wu H. Urine Albumin Excretion Is Associated with Cardiac Troponin $\mathrm{T}$ Detected with a Highly Sensitive Assay in a CommunityBased Population. PLoS One. 2015;10(8):e0135747.

37. Soga J, Nakamura S, Nishioka K, Umemura T, Jitsuiki D, Hidaka T, Teragawa H, et al. Relationship between augmentation index and flow-mediated vasodilation in the brachial artery. Hypertens Res. 2008;31(7):1293-1298.

38. McEvoy JW, Chen Y, Nambi V, Ballantyne CM, Sharrett AR, Appel LJ, Post WS, et al. High-Sensitivity Cardiac Troponin T and Risk of Hypertension. Circulation. 2015;132(9):825-833.

39. Hashimoto J, Watabe D, Hatanaka R, Hanasawa T, Metoki H, Asayama K, Ohkubo T, et al. Enhanced radial late systolic pressure augmentation in hypertensive patients with left ventricular hypertrophy. Am J Hypertens. 2006;19(1):27-32. 